\title{
Curcumin relieves paraquat-induced lung injury through inhibiting the thioredoxin interacting protein/NLR pyrin domain containing 3-mediated inflammatory pathway
}

\author{
YI REN, ZHIZHOU YANG, ZHAORUI SUN, WEI ZHANG, XIN CHEN and SHINAN NIE \\ Department of Emergency Medicine, Jinling Hospital, Medical School of Nanjing University, \\ Nanjing, Jiangsu 210002, P.R. China
}

Received July 19, 2018; Accepted May 30, 2019

DOI: $10.3892 / \mathrm{mmr} .2019 .10612$

\begin{abstract}
When paraquat (PQ) enters the human body, it increases oxidative stress and inflammation, ultimately resulting in acute lung injury (ALI). Curcumin, a naturally occurring compound, has been reported to ameliorate PQ-induced ALI; however, the underlying molecular mechanisms remain unclear. In the present study, normal lung fibroblasts (WI-38VA13) were treated with $10 \mu \mathrm{mol} / \mathrm{l} \mathrm{PQ}$ for $48 \mathrm{~h}$, followed by a further $48 \mathrm{~h}$ incubation with $300 \mu \mathrm{mol} / 1$ curcumin. Cells were then harvested to determine their viability. Flow cytometry was performed to analyze the levels of reactive oxygen species (ROS) and the rate of apoptosis. The levels of apoptotic proteins and activation of the thioredoxin interacting protein/NLR pyrin domain containing 3 (TXNIP/NLRP3) axis were measured via reverse transcription-quantitative polymerase chain reaction and western blot analyses. Proinflammatory cytokine levels were examined using enzyme-linked immunosorbent assays. Finally, the expression levels of Notch1, extracellular signal-regulated kinase 1/2 (ERK1/2) and phosphorylated-ERK1/2 were evaluated via western blotting. Following treatment with curcumin, PQ-induced increases in ROS levels and apoptosis were significantly attenuated, and $\mathrm{Bcl}-2$ expression levels were upregulated, whereas those of Bax were downregulated. It was also observed that curcumin treatment downregulated the expression levels of TXNIP, NLRP3, interleukin (IL)-1 $\beta$ and IL-18, and downstream caspase-1 compared with PQ treatment alone. Curcumin significantly attenuated the upregulation of Notch1 without affecting ERK1/2 phosphorylation. The present
\end{abstract}

Correspondence to: Dr Shinan Nie, Department of Emergency Medicine, Jinling Hospital, Medical School of Nanjing University, 305 East Zhongshan Road, Xuanwu, Nanjing, Jiangsu 210002, P.R. China

E-mail: shinann_niesn@163.com

Key words: paraquat, thioredoxin interacting protein/NLR pyrin domain containing 3 inflammasome axis, acute lung injury, curcumin, Notch1 signaling findings suggested that the inhibitory effects of curcumin on TXINP1 may inhibit activation of the NLRP3 inflammasome, subsequently suppressing the upregulation of proinflammatory cytokines and ultimately improving PQ-induced ALI.

\section{Introduction}

Paraquat (1,1'-dimethyl-4,4'-bipyridinium dichloride; PQ) is a widely used herbicide in agricultural production, as it possesses fast-acting and nonselective properties (1); however, the mortality rate following PQ poisoning is $>90 \%$, due to the lack of a specific antidote or effective therapy (2). It is known that the redox response is one of the initial factors involved in the toxic effects of PQ (3). Potent redox responses to PQ induce the production of a large quantity of reactive oxygen species (ROS), subsequently resulting in multiple organ injury via lipid peroxidation $(4,5)$. The lungs are usually one of the most damaged organs; accumulation of PQ in the lungs induces pulmonary edema, hemorrhage and alveolar epithelial cell destruction, ultimately leading to acute lung injury (ALI), one of the most frequent causes of PQ poisoning-associated mortality $(6,7)$. Numerous therapeutic strategies have been developed to target the pathological mechanisms of $\mathrm{PQ}$ poisoning; however, their clinical efficacies have not been satisfactory (8-10).

Thioredoxin (Trx) interacting protein (TXNIP) has been reported to interact with and inhibit Trx, which can modulate cellular ROS by reducing disulfides into thiol groups (11); however, under conditions of oxidative stress, the elevation of ROS levels induces the dissociation of TXINP from Trx, enabling Trx to contribute to ROS regulation (12). Of note, certain studies have demonstrated that TXNIP is involved in the activation of NLR pyrin domain containing 3 (NLRP3) (13). NLRP3, the best characterized inflammasome, consists of NLRP and the adaptor protein, apoptosis-associated speck-like protein containing caspase-1 activator domain (14). When the inflammasome is activated, caspase- 1 is cleaved to generate the active form of caspase-1, which in turn cleaves interleukin (IL)-1 $\beta$ and IL-18 into their active forms; these proinflammatory cytokines are subsequently involved in inflammation (15). Activation of the NLRP3 inflammasome has been demonstrated to serve an important role in 
PQ-induced lung damage (16). Therefore, NLRP3 may be a promising therapeutic target to treat PQ-induced ALI.

Curcumin $\left(\mathrm{C}_{21} \mathrm{H}_{20} \mathrm{O}_{6}\right)$ is a natural polyphenolic compound, which can be extracted from the powdered rhizome of the plant Curcuma longa L. Increasing evidence has indicated that curcumin possesses potent antioxidative and anti-inflammatory properties, with minimal side effects in humans (17-19). Notably, the inhibitory effects of curcumin on NLRP3-mediated inflammatory pathways have been reported; for example, Gong et al (20) demonstrated that curcumin effectively improves dextran sulfate sodium (DSS)-induced colitis in mice by inhibiting activation of the NLRP3 inflammasome; this study also reported that inhibiting the NLRP3 inflammasome in vivo with a specific NLRP3 inhibitor notably abrogates the additional inhibitory effects of curcumin on DSS-induced inflammatory bowel disease. Similarly, in fructose-induced hepatic inflammation, curcumin markedly inhibits NLRP3 inflammasome activation by upregulating microRNA-200a in mice, subsequently alleviating hepatic inflammation (21). Therefore, curcumin may be a promising drug for the treatment of NLRP3 inflammasome-associated inflammatory disease. Due to the central role of NLRP3 activation in PQ-induced ALI, the present study aimed to investigate whether curcumin could regulate activation of the NLRP3 inflammasome to improve PQ-induced lung damage.

\section{Materials and methods}

Cell culture. Normal lung fibroblasts [WI-38VA13; American Type Culture Collection (ATCC ${ }^{\circledR}$ ) CCL-75.1 ${ }^{\mathrm{TM}}$ ] were obtained from the ATCC. Cells were cultured in Minimum Essential Medium (MEM; ATCC ${ }^{\circledR} 30-2003^{\mathrm{TM}}$ ) and $10 \%$ fetal bovine serum (FBS; ATCC ${ }^{\circledR} 30-2020^{\mathrm{TM}}$; both ATCC) in a humidified atmosphere consisting of $5 \% \mathrm{CO}_{2}$ at $37^{\circ} \mathrm{C}$.

MTT assay. The effects of PQ or curcumin on the viability of WI-38VA13 cells were determined using an MTT assay (cat. no. M2128; Sigma-Aldrich; Merck KGaA) as previously described (22). Briefly, a single-cell suspension was incubated in 96-well plates at a cell density of $4 \times 10^{3}$ cells/well and supplemented with MEM containing 10\% FBS. To determine the appropriate experimental concentrations of curcumin and PQ, cells were treated with a series of concentrations of curcumin $(0,100,300$ and $600 \mu \mathrm{mol} / 1$; cat. no. 08511; Sigma-Aldrich; Merck KGaA) or PQ $(0,5,10$ and $20 \mu \mathrm{mol} / \mathrm{l}$; cat. no. 36541; Sigma-Aldrich; Merck $\mathrm{KGaA}$ ) for 0,24 or $48 \mathrm{~h}$ prior to cell viability detection. Subsequently, $10 \mu \mathrm{l}$ MTT solution $(5 \mathrm{mg} / \mathrm{ml})$ was added to each well, and the plates were incubated at $37^{\circ} \mathrm{C}$ for a further $4 \mathrm{~h}$. After removing the supernatant, DMSO (cat.no. D2650; Sigma-Aldrich; Merck KGaA) was added to dissolve the formazan crystals. The absorbance was detected at $450 \mathrm{~nm}$ using a microplate reader (Shanghai Cany Precision Instrument Co., Ltd.).

Effects of curcumin on PQ-induced WI-38VA13 cell injury. WI-38VA13 cells in the logarithmic growth phase were seeded in culture flasks, and were adjusted to a concentration of $1-2 \times 10^{4}$ cells $/ \mathrm{ml}$ in MEM (500 $\left.\mu \mathrm{l}\right)$. The following day, the medium was replaced with MEM containing $2 \%$ FBS. The cells were divided into four groups: Group 1 (Control) was treated with normal medium (MEM containing 2\% FBS) for
$96 \mathrm{~h}$; Group 2 (PQ) was exposed to PQ (10 $\mu \mathrm{mol} / \mathrm{l})$ for $48 \mathrm{~h}$ and then transferred to complete medium (MEM containing $10 \%$ FBS) for a further 48-h incubation; Group 3 (PQ + Cur) was exposed to PQ $(10 \mu \mathrm{mol} / \mathrm{l})$ for $48 \mathrm{~h}$, followed by treatment with $300 \mu \mathrm{mol} / 1$ curcumin for $48 \mathrm{~h}$; and Group 4 (Cur) was cultured with normal medium for $48 \mathrm{~h}$ and then treated with curcumin $(300 \mu \mathrm{mol} / \mathrm{l})$ for $48 \mathrm{~h}$. All groups were incubated in a humidified atmosphere consisting of $5 \% \mathrm{CO}_{2}$ at $37^{\circ} \mathrm{C}$. The cells were collected at three time points $(0,24$ and $48 \mathrm{~h})$ following completion of the $96 \mathrm{~h}$ treatment of cells for further MTT assays. Other subsequent experiments were conducted $\geq 48 \mathrm{~h}$ following completion of the 96 -h treatment of cells.

ROS detection assay. WI-38VA13 cells were seeded in 96 -well plates $\left(4 \times 10^{3}\right.$ cells/well) following various treatments. Following incubation for $48 \mathrm{~h}$, cells were trypsinized and harvested. A Reactive Oxygen Species Assay kit (Beyotime Institute of Biotechnology, cat. no. S0033) was used to determine the ROS levels. Briefly, cells were cultured with complete medium containing dichlorodihydrofluorescein diacetate at a final concentration of $10 \mu \mathrm{mol} / 1$ at $37^{\circ} \mathrm{C}$ for $20 \mathrm{~min}$. The cells were then harvested and washed with PBS at least three times. ROS levels were detected using a FACSCalibur flow cytometer (BD Biosciences), using BD CellQuest ${ }^{\mathrm{TM}}$ Pro Software version 5.1 (BD Biosciences).

Cell apoptosis analysis. Cells from each group were transferred to 96 -well plates $\left(4 \times 10^{3}\right.$ cells/well). Cells were incubated for $48 \mathrm{~h}$, trypsinized and harvested by centrifugation at 3,000 $\mathrm{x} \mathrm{g}$ for $5 \mathrm{~min}$ at $4^{\circ} \mathrm{C}$. The apoptosis rates were determined by Annexin-V/PI (propidine iodide) double-stain assay (BD Biosciences). The cells were resuspended in binding buffer containing $5 \mu \mathrm{l}$ Annexin V-FITC. Cells were stained in the dark for $15 \mathrm{~min}$ at room temperature. Subsequently, $5 \mu \mathrm{PI}$ was added $5 \mathrm{~min}$ before the assay, and the apoptotic rates of the treated WI-38VA13 cells were determined using a FACS Calibur flow cytometer (BD Biosciences), using BD CellQuest $^{\mathrm{TM}}$ Pro Software version 5.1 (BD Biosciences).

Reverse transcription-quantitative polymerase chain reaction $(R T-q P C R)$. Total RNA was extracted from treated WI-38VA13 cells using TRIzol ${ }^{\circledR}$ reagent (Invitrogen; Thermo Fisher Scientific, Inc.) and was then purified using RNase-free DNase (Takara Biotechnology Co., Ltd.). A PrimeScript ${ }^{\mathrm{TM}}$ First Strand cDNA Synthesis kit (Takara Biotechnology Co., Ltd.) was used to synthesize cDNA as previously described (23) under the following conditions: $65^{\circ} \mathrm{C}$ for $5 \mathrm{~min}, 30^{\circ} \mathrm{C}$ for $6 \mathrm{~min}$ and $50^{\circ} \mathrm{C}$ for $50 \mathrm{~min}$. Sequences of the specific primers are presented in Table I. An ABI 7500 Fast Real-Time PCR system (Applied Biosystems; Thermo Fisher Scientific, Inc.) was used to perform qPCR reactions using a reaction mixture containing $1 \mu \mathrm{l}$ forward and reverse primers (10 $\mu \mathrm{mol} / \mathrm{l}), 10 \mu \mathrm{l}$ SYBR fluorescent dye (Applied Biosystems; Thermo Fisher Scientific, Inc.), $2 \mu \mathrm{l}$ cDNA and RNase-free $\mathrm{dH}_{2} \mathrm{O}$. The thermocycling parameters were as follows: Initial denaturation at $94^{\circ} \mathrm{C}$ for $3 \mathrm{~min}$, followed by repeated amplification for 40 cycles at $94^{\circ} \mathrm{C}$ for $30 \mathrm{sec}, 56^{\circ} \mathrm{C}$ for $30 \mathrm{sec}$ and $72^{\circ} \mathrm{C}$ for $2 \mathrm{~min}$, prior to a final step at $72^{\circ} \mathrm{C}$ for $10 \mathrm{~min}$. GAPDH served as an internal control. The relative mRNA expression levels were calculated using the $2^{-\Delta \Delta \mathrm{Cq}}$ method (24). 
Enzyme-linked immunosorbent assay (ELISA). The treated WI-38VA13 cells were collected by centrifugation at $300 \mathrm{x}$ g for $7 \mathrm{~min}$ at $4^{\circ} \mathrm{C}$. After washing with PBS, the cells were lysed using cell extraction buffer (cat. no. FNN0011; Thermo Fisher Scientific, Inc.) with PMSF (1 mM) and protease inhibitor cocktail (cat. no. P-2714; Sigma-Aldrich; Merck KGaA). Subsequently, the mixture was incubated at $4^{\circ} \mathrm{C}$ for $30 \mathrm{~min}$. After centrifuging the cells at $13,000 \mathrm{x} \mathrm{g}$ for $10 \mathrm{~min}$ at $4^{\circ} \mathrm{C}$, the supernatant was removed. IL-1 $\beta$ and IL-18 levels were determined using IL- $1 \beta$ and IL-18 assay kits (cat. nos. H002 and H015; Nanjing Jiancheng Bioengineering Institute Co., Ltd.), according to the manufacturer's protocols. The primary IL-1 $\beta$ and IL-18 primary antibodies were added to the wells. The prepared standard liquid and diluted supernatant (1:4) were added to ELISA plates. The horseradish peroxidase (HRP)-labeled secondary antibodies were added to each well, and then the plate was incubated in a humidified box at $37^{\circ} \mathrm{C}$ for $60 \mathrm{~min}$. After the solution was discarded, substrate solution was added to each well, and the optical density was measured using a spectrophotometer (cat. no. 722S; Shanghai Precision \& Scientific Instrument Co., Ltd.).

Western blotting. Total protein was extracted from WI-38VA13 cells using RIPA solution (Sigma-Aldrich; Merck KGaA), and then quantified via a bicinchoninic acid protein assay (Takara Biotechnology Co., Ltd.). Equal quantities of total protein $(30 \mu \mathrm{g})$ were separated by $12 \%$ SDS-PAGE and then transferred to PVDF membranes (EMD Millipore). After blocking in 5\% non-fat milk in TBS-Tween for $1 \mathrm{~h}$ at room temperature, membranes were incubated at $4^{\circ} \mathrm{C}$ overnight with the following primary antibodies (all from Cell Signaling Technology, Inc.): Anti-Bcl-2 (rabbit; 28 kDa; 1:1,000; cat. no. 2872); anti-Bax (rabbit; $20 \mathrm{kDa}$; 1:1,000; cat. no. 2774); anti-cleaved caspase-1 (rabbit; $20 \mathrm{kDa} ; 1: 1,000$; cat. no. 4199); anti-caspase-1 (rabbit; $48 \mathrm{kDa}$; 1:1,000; cat. no. 3866); anti-NLRP3 (rabbit; $110 \mathrm{kDa}$; 1:1,000; cat. no. 15101), anti-TXINP (rabbit; 55 kDa; 1:1,000; cat. no. 14715); anti-Notch1 (rabbit; 120 kDa; 1:1,000; cat. no. 4380), anti-phosphorylated (p)-extracellular signal-regulated kinase 1/2 (p-ERK1/2; rabbit; 42 and $44 \mathrm{kDa}$; 1:1,000; cat. no. 9101); anti-ERK1/2 (rabbit; 42 and $44 \mathrm{kDa}$; 1:1,000; cat. no. 9102); and anti-GAPDH (rabbit; $37 \mathrm{kDa}$; 1:1,000; cat. no. 5174). Subsequently, membranes were incubated with HRP-conjugated goat anti-rabbit immunoglobulin G secondary antibodies (1:2,000; cat. no. 7074; Cell Signaling Technology, Inc.) for $2 \mathrm{~h}$ at room temperature. Proteins were detected using Pierce ${ }^{\mathrm{TM}}$ ECL western blotting substrate (cat. no. 32106; Thermo Fisher Scientific, Inc.). Optical band density was semi-quantified using Bio-Rad ChemiDoc ${ }^{\mathrm{TM}}$ XRS+ System with Image Lab ${ }^{\mathrm{TM}}$ Software version 4.1 (Bio-Rad Laboratories, Inc.). GAPDH was used as an internal control.

Statistical analysis. Statistically significant differences among three or more groups were analyzed by one-way analysis of variance followed by Tukey's post hoc test, using SPSS version 20 (IBM Corp.). Each experiment was repeated three times. All data were presented as the mean \pm standard error of the mean. $\mathrm{P}<0.05$ was considered to indicate a statistically significant difference.
Table I. Primers for reverse transcription-quantitative polymerase chain reaction.

\section{Gene \\ name Primer sequences

\begin{tabular}{ll}
\hline Bax & Forward: 5'-GATGCGTCCACCAAGAAG-3' \\
& Reverse: 5'-AGTTGAAGTTGCCGTCAG-3' \\
Bcl-2 & Forward: 5'-GTTCCCTTTCCTCCATCC-3' \\
& Reverse: 5'-TAGCCAGTCCAGAGGTGAG-3'
\end{tabular} \\ GAPDH Forward: 5'-ATGGCACCGTCAAGGCTGAG-3' \\ Reverse: 5'-TGTCAGGTACGGTAGTGACG-3' \\ TXNIP Forward: 5'-GCTCAATCATGGTGATGTTCA AG-3' \\ Reverse: 5'-CTTCACACACTTCCACTGTCAC-3' \\ NLRP3 Forward: 5'-GGAGAGACCTTTATGAGAAAGC AA-3' \\ Reverse: 5'-GCTGTCTTCCTGGCATATCACA-3' \\ Notch1 Forward: 5'-CACCCATGACCACTACCCAGTT-3' Reverse: 5'-CCTCGGACCAATCAGAGATGTT-3'}

NLRP3, NLR pyrin domain containing 3; TXNIP, thioredoxin interacting protein.

\section{Results}

Curcumin mitigates PQ-induced apoptosis of WI-38VA13 cells. The viability of WI-38VA13 cells was detected at 24 and $48 \mathrm{~h}$. As shown in Fig. 1A, cell viability was unchanged following treatment with $300 \mu \mathrm{mol} / 1$ curcumin; however, a decrease in viability was observed in response to treatment with $600 \mu \mathrm{mol} / 1$ curcumin for $48 \mathrm{~h}$ compared with in the control group. In addition, the effects of $10 \mu \mathrm{mol} / 1 \mathrm{PQ}$ on cell viability were more noticeable compared with $5 \mu \mathrm{mol} / 1 \mathrm{PQ}$ (Fig. 1B). Therefore, $10 \mu \mathrm{mol} / 1 \mathrm{PQ}$ was selected to induce lung cell injury and the positive effects of $300 \mu \mathrm{mol} / 1$ curcumin were assessed on the toxicity of PQ. As shown in Fig. 2A, the viability of cells treated with PQ was significantly reduced compared with in the control group, whereas the cell viability of the PQ + Cur group was higher than that of the PQ group. In addition, ROS levels and apoptosis were measured by flow cytometry. As predicted, treatment with PQ promoted the production of ROS and significantly increased apoptosis (Fig. 2B and C). As shown in Fig. 2B-E, PQ treatment induced ROS generation and increased the percentage of apoptotic cells from 4.50 to $24.68 \%(\mathrm{P}<0.01)$ compared with in the control group. Conversely, the apoptosis of cells treated with curcumin alone was no different from that in the control group. Furthermore, compared with in the PQ-treated group, the ROS levels were significantly reduced in the $\mathrm{PQ}+$ Cur group $(\mathrm{P}<0.05)$, and the percentage of apoptosis fell to $10.92 \%$. These results indicated that curcumin may be able to greatly reduce PQ-induced ROS production and apoptosis; however, curcumin could not completely protect lung cells against PQ-induced damage.

Curcumin suppresses apoptosis-associated protein expression in PQ-damaged WI-38VAl3 cells. The expression levels of the apoptosis-associated proteins Bcl-2 and Bax 



Figure 1. Effects of curcumin and PQ on cell viability. To determine appropriate experimental concentrations of curcumin and PQ, cells were treated with a series of curcumin $(0-600 \mu \mathrm{mol} / \mathrm{l})$ or PQ $(0-20 \mu \mathrm{mol} / \mathrm{l})$ concentrations. (A) WI-38VA13 cells were treated with different concentrations of curcumin (100, 300 and $600 \mu \mathrm{mol} / \mathrm{l})$. (B) WI-38VA13 cells were treated with different concentrations of PQ $(5,10$ and $20 \mu \mathrm{mol} / \mathrm{l})$. Data are presented as the mean \pm standard error of the mean $(\mathrm{n}=3) .{ }^{*} \mathrm{P}<0.05$ and ${ }^{* *} \mathrm{P}<0.01$ vs. 0 h. Cur, curcumin; OD, optical density; PQ, paraquat.
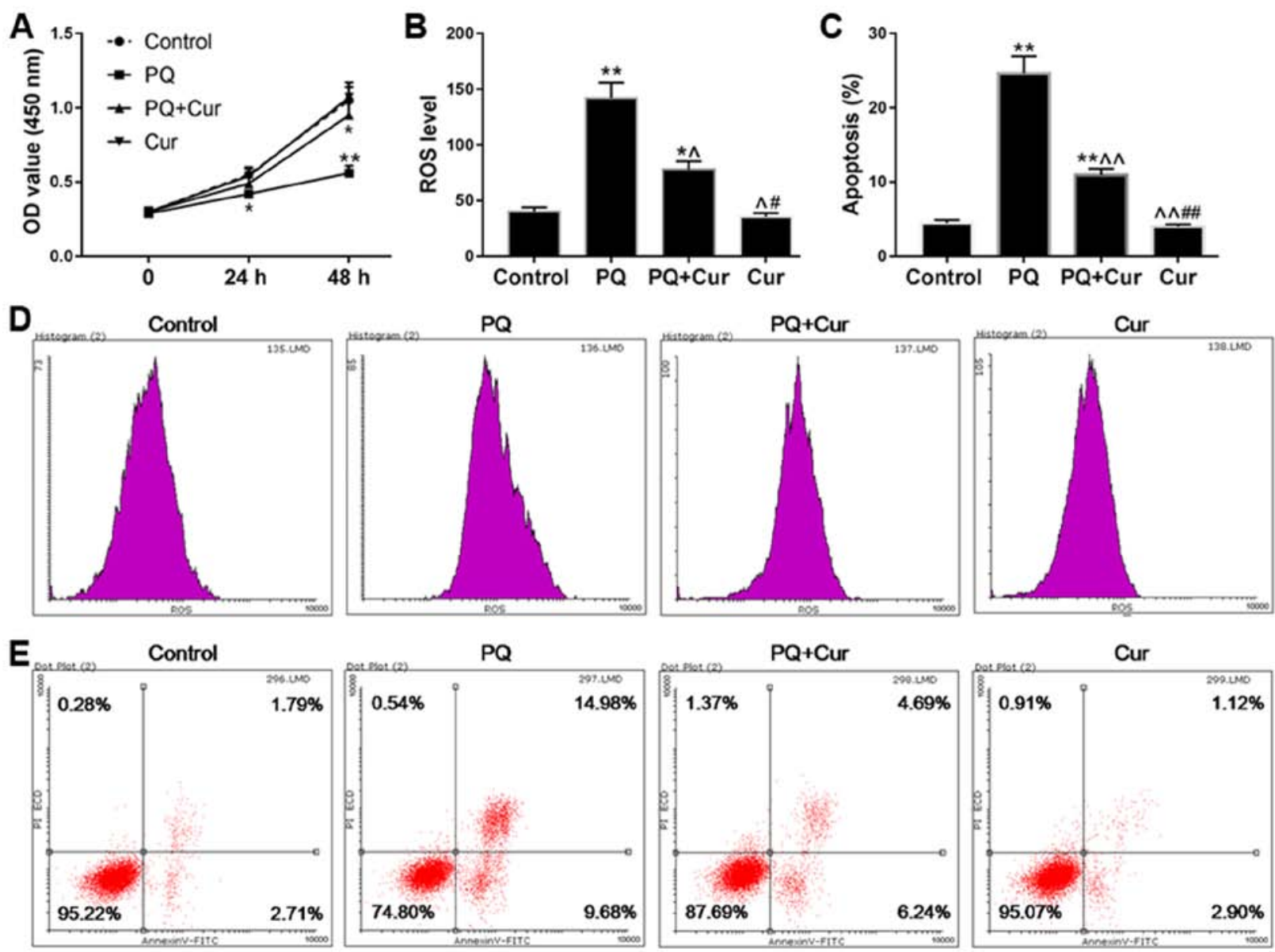

Figure 2. Curcumin reduces ROS levels and apoptosis in PQ-treated WI-38VA13 cells. PQ $(10 \mu \mathrm{mol} / \mathrm{l})$ and curcumin $(300 \mu \mathrm{mol} / \mathrm{l})$ were selected as the experimental concentrations for subsequent experiments. (A) Curcumin enhanced the viability of PQ-damaged WI-38VA13 cells. (B and D) Effects of PQ and curcumin on ROS levels. (C and E) Effects of PQ and curcumin on the apoptosis of WI-38VA13 cells. Data are presented as the mean \pm standard error of the mean (n=3). ${ }^{*} \mathrm{P}<0.05,{ }^{* *} \mathrm{P}<0.01$ vs. control; ${ }^{\wedge} \mathrm{P}<0.05,{ }^{\wedge} \mathrm{P}<0.01$ vs. $\mathrm{PQ}$; ${ }^{\#} \mathrm{P}<0.05,{ }^{\# \#} \mathrm{P}<0.01$ vs. $\mathrm{PQ}+$ Cur. Cur, curcumin; OD, optical density; PI, propidium iodide; $\mathrm{PQ}$, paraquat; ROS, reactive oxygen species.

in WI-38VA13 cells were determined via western blotting and RT-qPCR. Significantly upregulated Bax mRNA and protein levels were detected following treatment of cells with $10 \mu \mathrm{mol} / 1 \mathrm{PQ}$ for $48 \mathrm{~h}$ compared with the control; however, treatment with $\mathrm{PQ}+$ Cur significantly decreased the expression levels of Bax (Fig. 3A-C). Bcl-2, an anti-apoptotic protein, was significantly downregulated by $\mathrm{PQ}$, whereas curcumin inhibited these effects and significantly increased the expression of Bcl-2 (Fig. 3A-C). Conversely, compared with the effects of curcumin on the protein expression levels of Bax and Bcl-2 in PQ-damaged cells (PQ + Cur group), WI-38VA13 cells treated with curcumin alone (Cur group) exhibited a more upregulated Bcl-2 levels and downregulated Bax levels. 

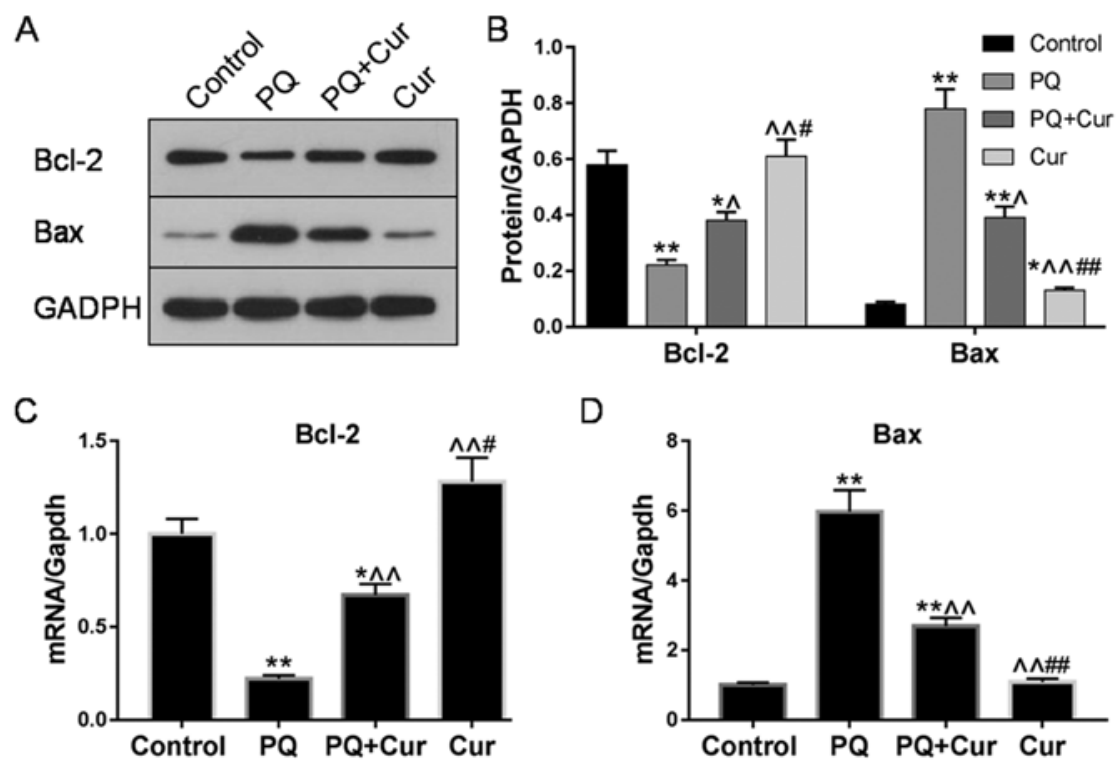

Figure 3. Curcumin suppresses apoptosis-associated protein expression in PQ-damaged WI-38VA13 cells. WI-38VA13 cells were treated with PQ (10 $\mu$ mol/l) for $48 \mathrm{~h}$, followed by a further 48-h incubation with curcumin $(300 \mu \mathrm{mol} / \mathrm{l})$. (A-D) Expression levels of Bcl-2 and Bax as determined by western blotting and reverse transcription-quantitative polymerase chain reaction. GAPDH was used as an internal control. Data are presented as the mean \pm standard error of the mean $(\mathrm{n}=3)$. ${ }^{*} \mathrm{P}<0.05,{ }^{* *} \mathrm{P}<0.01$ vs. control; ${ }^{\wedge} \mathrm{P}<0.05,{ }^{\wedge} \mathrm{P}<0.01$ vs. $\mathrm{PQ} ;{ }^{\#} \mathrm{P}<0.05,{ }^{\#} \mathrm{P}<0.01$ vs. $\mathrm{PQ}+$ Cur. Cur, curcumin; $\mathrm{PQ}$, paraquat.
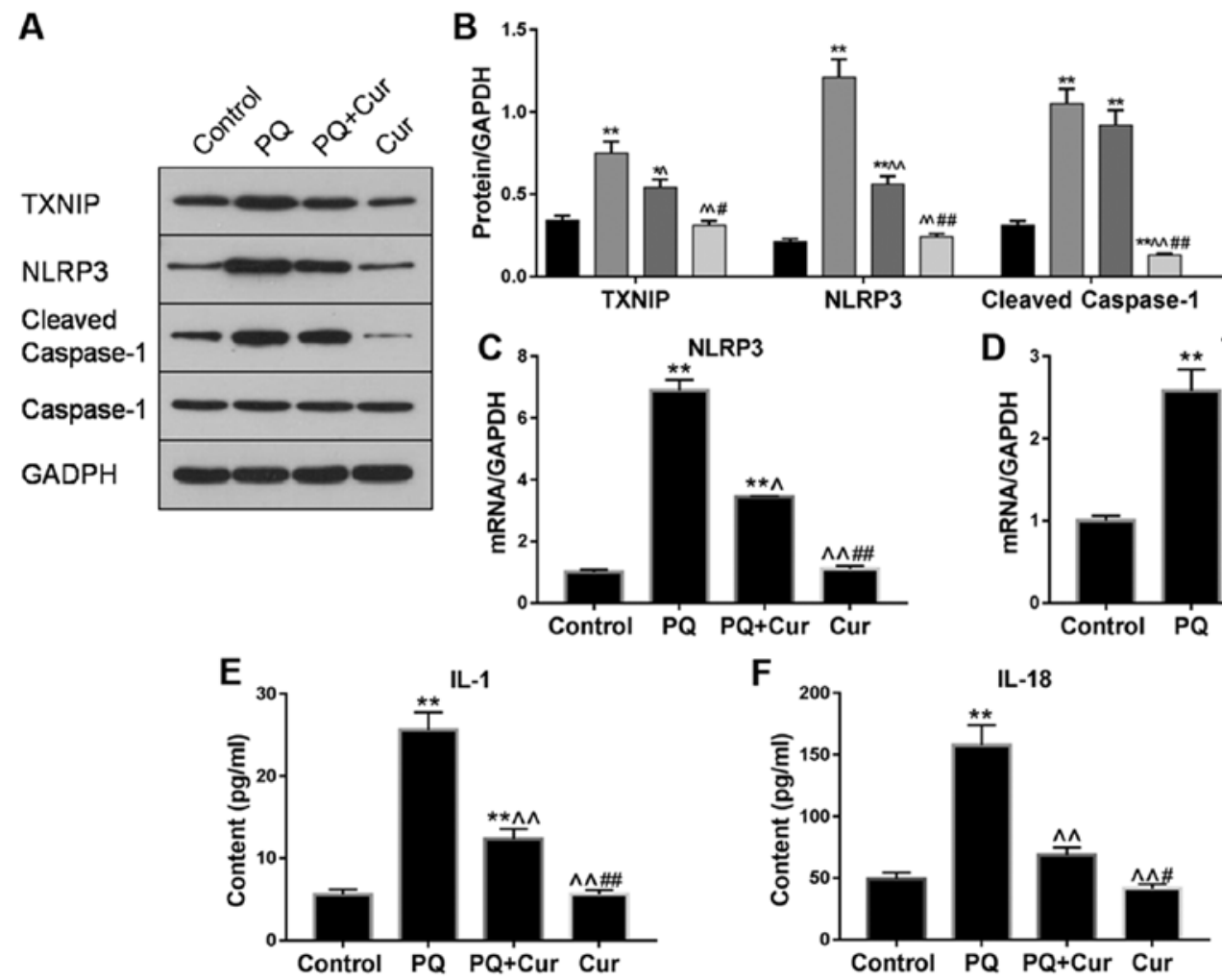

Figure 4. Curcumin inhibits the TXNIP/NLRP3 axis and proinflammatory cytokine release in PQ-treated WI-38VA13 cells. PQ (10 $\mu$ mol/l) and curcumin ( $300 \mu \mathrm{mol} / \mathrm{l}$ ) were used to study the mechanisms underlying the effects of curcumin on PQ-induced lung cell injury. (A and B) Protein levels of TXNIP, NLRP3, cleaved caspase-1 and caspase-1 as determined via western blotting. mRNA levels of (C) NLRP3 and (D) TXNIP were detected via reverse transcription-quantitative polymerase chain reaction. GAPDH was used as an internal control. Levels of (E) IL-1 $\beta$ and (F) IL-18 were quantified by enzyme-linked immunosorbent assay. Data are presented as the mean \pm standard error of the mean $(\mathrm{n}=3) .{ }^{*} \mathrm{P}<0.05,{ }^{* *} \mathrm{P}<0.01$ vs. control; ${ }^{\wedge} \mathrm{P}<0.05,{ }^{\wedge} \mathrm{P}<0.01$ vs. $\mathrm{PQ} ;{ }^{*} \mathrm{P}<0.05,{ }^{\# \#} \mathrm{P}<0.01 \mathrm{vs}$. $\mathrm{PQ}+$ Cur. Cur, curcumin; IL, interleukin; NLRP3, NLR pyrin domain containing 3; PQ, paraquat; TXNIP, thioredoxin interacting protein.

Curcumin inhibits the activated TXNIP/NLRP3 axis in cultured $P Q$-treated WI-38VAl3 cells. An increasing number of studies have reported that inflammation serves an important role in lung injury $(25,26)$. To investigate whether curcumin reduced lung inflammation induced by PQ, the expression levels of TXNIP, NLRP3, cleaved caspase-1 and caspase-1 were evaluated by western blotting and RT-qPCR. As presented in Fig. 4A-D, significant upregulation of TXNIP, NLRP3 and cleaved 
A
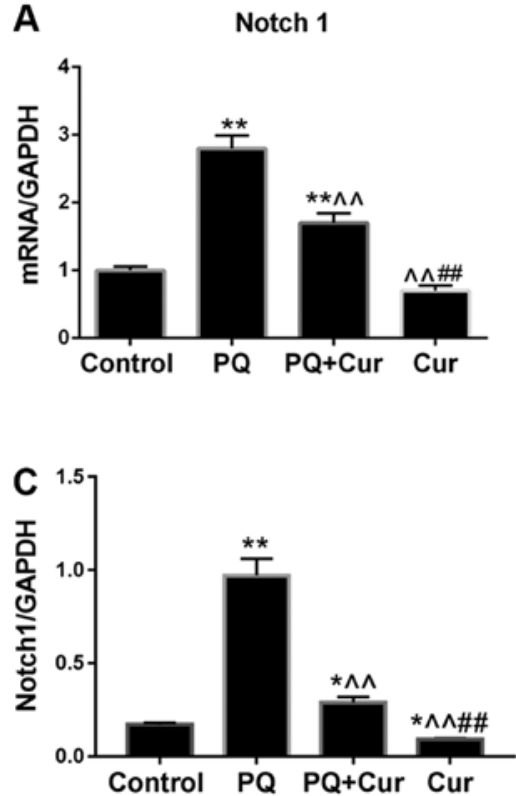

B


Figure 5. Curcumin modulates signaling pathways in PQ-treated WI-38VA13 cells. (A) Relative mRNA expression levels of Notch1 as determined by reverse transcription-quantitative polymerase chain reaction. (B) Protein expression levels of Notch1, p-ERK1/2 and ERK1/2 as detected via western blotting (C) Protein expression levels of Notch1. (D) Ratio of p-ERK1/2 to ERK1/2. GAPDH was used as an internal control. Data are presented as the mean \pm standard error of the mean $(\mathrm{n}=3)$. * $\mathrm{P}<0.05,{ }^{* *} \mathrm{P}<0.01$ vs. control; ${ }^{\wedge} \mathrm{P}<0.01$ vs. $\mathrm{PQ}$; ${ }^{\# \#} \mathrm{P}<0.01$ vs. $\mathrm{PQ}+$ Cur. Cur, curcumin; ERK1/2, extracellular signal-regulated kinase $1 / 2$; p, phosphorylated; PQ, paraquat.

caspase-1 was observed in the PQ-treated group compared with the control; however, there was no significant change observed in caspase- 1 expression. Additionally, following treatment of lung cells with curcumin, the mRNA and protein expression levels of TXNIP and NLRP3 were significantly decreased compared with PQ treatment alone, whereas the effects on cleaved caspase-1 levels were only slightly attenuated.

To further determine whether curcumin affected PQ-induced activation of proinflammatory cytokines, the levels of IL-1 $\beta$ and IL-18 in cell extracts were determined by ELISA. It was revealed that PQ treatment alone significantly increased IL-1 $\beta$ and IL-18 levels compared with the control; however, treatment with curcumin significantly suppressed the elevated levels of these proinflammatory cytokines (Fig. 4E). Collectively, these results demonstrated that curcumin suppressed PQ-induced NLRP3 activation and downstream inflammatory pathways.

Involvement of signaling pathways in the effects of curcumin on PQ-induced WI-38VA13 cell injury. As presented in Fig. 5A-C, PQ treatment significantly upregulated the mRNA and protein expression levels of Notch1, whereas they were significantly reduced by curcumin. Additionally, the levels of p-ERK1/2 and ERK1/2 were assessed by western blotting (Fig. 5B). The ratio of p-ERK1/2 to total ERK1/2 for each group is presented in Fig. 5D. Significant increases in Notch1 and p-ERK1/2/ERK1/2 levels were observed in the PQ group compared with the control (Fig. 5C and D). Curcumin treatment significantly attenuated the PQ-induced upregulation of Notch1; however, no significant difference in p-ERK1/2/ERK1/2 was observed compared with PQ treatment alone. Of note, significantly increased phosphorylation of ERK1/2 was also observed following treatment with curcumin alone compared with the control.

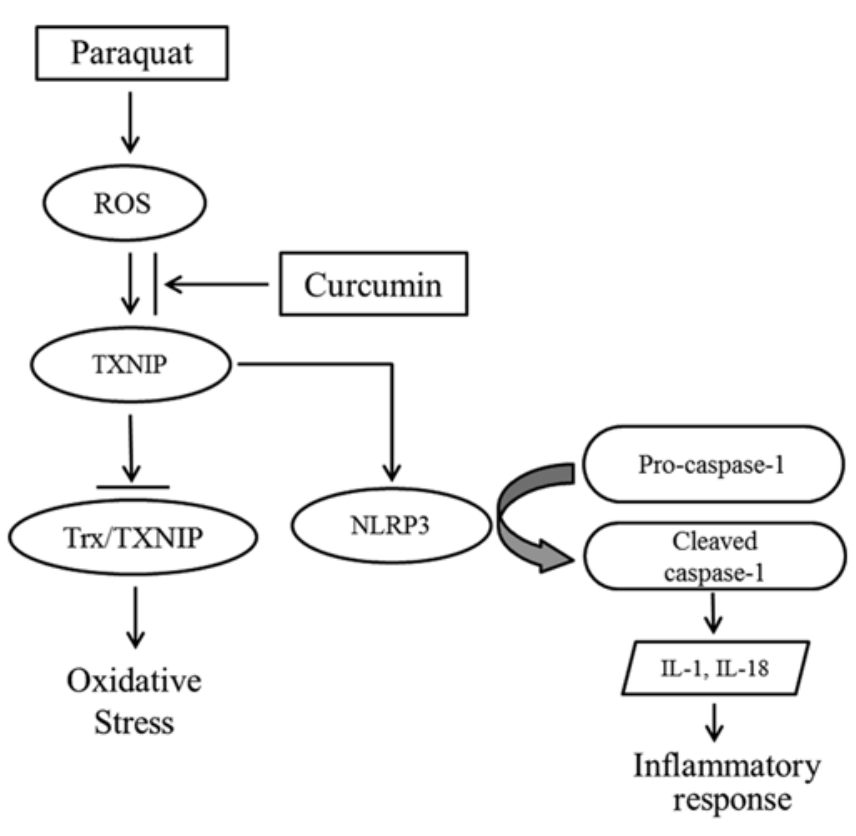

Figure 6. Schematic illustration of the proposed mechanisms underlying the effects of curcumin on PQ-induced oxidative stress and inflammatory response. PQ induces excessive production of ROS, which in turn promotes the expression of TXNIP. TXINIP not only interacts with Trx and blocks its antioxidative ability, but also activates the NLRP3-mediated inflammatory pathway. Curcumin is proposed to inhibit the promoting effects of ROS. IL, interleukin; NLRP3, NLR pyrin domain containing 3; PQ, paraquat; ROS, reactive oxygen species; Trx, thioredoxin; TXNIP, Trx interacting protein.

\section{Discussion}

The toxic effects of PQ are primarily mediated via oxidative stress and inflammatory responses (27) In 2014, Tyagi et al (28) reported that intranasal curcumin directly targets the lungs of mice, and 
significantly improves PQ-induced lung damage by inhibiting the production of proinflammatory cytokines and ROS. In the present study, it was revealed that treatment with curcumin significantly attenuated PQ-induced elevated ROS levels and apoptosis in WI-38VA13 cells. Additionally, the expression of proteins in the TXNIP/NLRP3-mediated proinflammatory pathway was determined, and it was demonstrated that curcumin also significantly decreased activation of the NLRP3 inflammasome. Furthermore, downregulation of Notch1 was observed, indicating that the Notch signaling pathway may serve a potential role in the mechanisms underlying the effects of curcumin on PQ-induced ALI. Collectively, the present findings suggested that curcumin may aid in relieving oxidative stress and inflammation induced by PQ, and that these effects may be mediated by decreasing ROS levels and inhibiting the activation of NLRP3.

Previous studies have reported that PQ poisoning leads to the depletion of cellular nicotinamide adenine dinucleotide phosphate by generating large quantities of ROS, which potently promotes oxidative stress and inflammatory responses, and that the increasing lipid peroxidation may alter the function of cell membrane receptors, inducing immune cells to produce ILs and other inflammatory cytokines (28-30). The Trx/TXNIP complex has been revealed to be an important regulator of cellular redox status; overproduction of ROS induces the separation of Trx and TXNIP, after which Trx promotes the biosynthesis of manganese superoxide dismutase to inhibit ROS production $(31,32)$, whereas TXNIP directly interacts with NLRP3 and promotes activation of the NLRP3 inflammasome (13). In 2016, Kim et al (3) demonstrated that ROS exhibits the ability to induce upregulated TXNIP expression. In the present study, it was demonstrated that the expression of TXNIP was significantly increased following PQ treatment. In addition to affecting the Trx/TXNIP complex, ROS have also been proposed to directly activate NLRP3 $(33,34)$. The NLRP3 inflammasome serves an important role in inflammation and innate immunity, and has been reported to be involved in the pathogenesis of various types of inflammatory diseases (35-37), including PQ-induced ALI (16). Considering the association between TXNIP and NLRP3, it was hypothesized that the mechanisms underlying PQ-induced inflammatory responses in the lung may be mediated by the promoting effects of ROS on TXNIP expression and subsequent NLRP3 activation. In the present study, treatment with curcumin significantly reduced ROS generation, which may suppress ROS-mediated activation of the TXNIP/NLRP3 inflammatory pathway, and downstream caspase- 1 cleavage, and IL-1 $\beta$ and IL-18 secretion. Furthermore, it was observed that the apoptosis of WI-38VA13 cells was significantly increased by treatment with PQ. Previous studies revealed that ROS-associated apoptosis is mainly associated with the apoptosis-signal kinase 1/Trx/TXNIP and TXNIP/NLRP3 inflammasome signaling pathways $(38,39)$. According to the results of flow cytometry, and the expression of the anti-apoptotic protein Bcl-2 and proapoptotic protein $\mathrm{Bax}$, the present results suggested that curcumin inhibited PQ-induced apoptosis and inflammation in WI-38VA13 cells, potentially by reducing ROS generation.

The protein levels of Notch1, p-ERK1/2 and ERK1/2 were also investigated by western blotting. Previous studies have indicated that activation of Notch signaling promotes tissue inflammation, neoplasia and metastasis $(40,41)$. In the present study, significant upregulation of Notch1 was observed in PQ-treated WI-38VA13 cells. In 2014, Singla et al (42) confirmed that increased Notch1 levels promote proinflammatory cytokine release by activating macrophage differentiation, whereas inhibiting Notch1 expression or blocking the Notch signaling pathway decreases proinflammatory cytokine production and secretion. The present findings suggested that curcumin downregulated Notch1 signaling, potentially subsequently attenuating the synthesis and release of proinflammatory cytokines; however, Jiang et al (43) reported that Notch signaling induces inhibitory effects on NLRP3 activation by suppressing the high mobility group protein B1/Toll-like receptor $4 / \mathrm{NF}-\kappa \mathrm{B}$ pathway in acetaminophen-induced liver injury, in contrast with the present study. It was also observed that PQ induced upregulation of $\mathrm{p}-\mathrm{ERK} 1 / 2$; however, curcumin treatment did not significantly affect PQ-induced phosphorylation, indicating that ERK1/2 signaling may be involved in the mechanisms underlying PQ-induced ALI but not those contributing towards the protective effects of curcumin. Collectively, Notch signaling may serve a role in the effects of curcumin on PQ-induced ALI; however, the underlying molecular mechanisms require further investigation.

The present study possessed certain limitations. Additional inflammatory cytokines should be measured to more fully characterize the activation of TXNIP/NLRP3-mediated inflammatory pathways. In addition, the mechanisms underlying the potential involvement of the Notch pathway in the effects of curcumin on PQ-induced ALI are yet to be investigated.

In conclusion, the present study suggested that $\mathrm{PQ}$ poisoning induced the overproduction of ROS, which may promote TXNIP expression. TXNIP not only inhibits the antioxidative ability of Trx, but also contributes to NLRP3 activation, and the maturation and secretion of proinflammatory cytokines (13). Following treatment with curcumin, inflammation and apoptosis were significantly decreased, and protein expression analysis suggested that the beneficial effects of curcumin may be mediated by suppressing the TXNIP/NLRP3 inflammatory axis (Fig. 6). In addition, it was observed that Notch1 signaling served a potential role in the mechanisms via which curcumin attenuated PQ poisoning. The present findings provide further understanding of the molecular mechanisms underlying the effects of curcumin on PQ-induced lung injury, and indicated that TXNIP/NLRP3 may be a novel therapeutic target for the treatment of PQ-induced ALI.

\section{Acknowledgements}

Not applicable.

\section{Funding}

The present study was supported by the National Natural Science Foundation of China (grant nos. 81401583 and 81701894), Social Development Projects of Jiangsu Province (grant no. BE2017720), Jiangsu Provincial Medical Youth Talent (grant nos. QNRC2016908 and QNRC2016909), the Major Projects Foundation of General Logistics Department of PLA (grant no. CNJ14L002), the Peking Union Farsighted Emergency Project (grant no. RE2016002) and the Natural Science Foundation of Jinling Hospital (grant nos. 2015027 and 2016032). 


\section{Availability of data and materials}

The datasets used and/or analyzed during the current study are available from the corresponding author on reasonable request.

\section{Authors' contributions}

$\mathrm{XC}$ and SN conceived and designed the study. YR studied the effect of curcumin on ROS and apoptosis induced by paraquat. ZY studied the effect of curcumin on apoptosis related factors induced by paraquat. ZS studied the effect of curcumin on Notch pathway activated by paraquat. WZ studied the effect of curcumin on proinflammatory cytokines induced by paraquat. $\mathrm{SN}$ was a major contributor in writing the manuscript. All authors read and approved the final manuscript.

\section{Ethics approval and consent to participate}

Not applicable.

\section{Patient consent for publication}

Not applicable.

\section{Competing interests}

The authors declare that they have no competing interests.

\section{References}

1. Baltazar T, Dinis-Oliveira RJ, Duarte JA, de Lourdes Bastos M and Carvalho F: Paraquat research: Do recent advances in limiting its toxicity make its use safer? Br J Pharmacol 168: 44-45, 2013.

2. Zhang B, Lang Y and Yi O: Literature analysis of paraquat poisoning in China (1991-2008). Chin J Crit Care Med 30: 139-141, 2010 (In Chinese).

3. Kim E, Leverage WT, Liu Y, Panzella L, Alfieri ML, Napolitano A, Bentley WE and Payne GF: Paraquat-melanin redox-cycling: Evidence from electrochemical reverse engineering. ACS Chem Neurosci 7: 1057-1067, 2016.

4. Dinis-Oliveira RJ, Duarte JA, Sanchez-Navarro A, Remião F, Bastos ML and Carvalho F: Paraquat poisonings: Mechanisms of lung toxicity, clinical features, and treatment. Crit Rev Toxicol 38: 13-71, 2008.

5. Tomita M, Okuyama T, Katsuyama H, Miura Y, Nishimura Y, Hidaka K, Otsuki $\mathrm{T}$ and Ishikawa $\mathrm{T}$ : Mouse model of paraquat-poisoned lungs and its gene expression profile. Toxicology 231: 200-209, 2007.

6. Liu J, Xiong Y and Jiang M: Ratio of injured lung volume fraction in prognosis evaluation of acute PQ poisoning. Biomed Res Int 2018: e4501536, 2018.

7. Yang W, Liu W, Yu W, Fei D, Meng X, Yang S, Meng S and Zhao M: Angptl2 deficiency attenuates paraquat (PQ)-induced lung injury in mice by altering inflammation, oxidative stress and fibrosis through NF- $\kappa$ B. Biochem Biophys Res Commun 503: 94-101, 2018

8. Hu S, Qiao C, Yuan Z, Li M, Ye J, Ma H, Wang J, Xin S and Zhang J: Therapy with high-dose long-term antioxidant free radicals for severe paraquat poisoning: A pilot study. Exp Ther Med 16: 5149-5155, 2018

9. Buendia JA, Chavarriaga GJR and Zuluaga AF: Burden of paraquat poisoning in the department of Antioquia, Colombia. BMC Pharmacol Toxicol 20: 11, 2019.

10. Nasr Isfahani S, Farajzadegan Z, Sabzghabaee AM, Rahimi A, Samasamshariat S and Eizadi-Mood N: Does hemoperfusion in combination with other treatments reduce the mortality of patients with paraquat poisoning more than hemoperfusion alone: A systematic review with meta-analysis. J Res Med Sci 24: 2, 2019.
11. Devi TS, Lee I, Hüttemann M, Kumar A, Nantwi KD and Singh LP: TXNIP links innate host defense mechanisms to oxidative stress and inflammation in retinal Muller glia under chronic hyperglycemia: Implications for diabetic retinopathy. Exp Diabetes Res 2012: 438238, 2012.

12. Yoshihara E, Masaki S, Matsuo Y, Chen Z, Tian H and Yodoi J: Thioredoxin/Txnip: Redoxisome, as a redox switch for the pathogenesis of diseases. Front Immunol 4: 514, 2014.

13. Zhou R, Tardivel A, Thorens B, Choi I and Tschopp J: Thioredoxin-interacting protein links oxidative stress to inflammasome activation. Nat Immunol 11: 136-140, 2010.

14. Lamkanfi $M$ and Dixit VM: Inflammasomes and their roles in health and disease. Annu Rev Cell Dev Biol 28: 137-161, 2012.

15. Schroder K and Tschopp J: The inflammasomes. Cell 140: 821-832, 2010.

16. Liu Z, Zhao H, Liu W, Li T, Wang Y and Zhao M: NLRP3 inflammasome activation is essential for paraquat-induced acute lung injury. Inflammation 38: 433-444, 2015.

17. Narayanan A, Kehn-Hall K, Senina S, Lundberg L, Van Duyne R, Guendel I, Das R, Baer A, Bethel L, Turell M, et al: Curcumin inhibits rift valley fever virus replication in human cells. J Biol Chem 287: 33198-33214, 2012.

18. Rahmani AH, AlZohairy MA, Aly SM and Khan MA: Curcumin: A potential candidate in prevention of cancer via modulation of molecular pathways. Biomed Res Int 2014: 761608, 2014.

19. Shehzad A, Lee J and Lee YS: Curcumin in various cancers. Biofactors 39: 56-68, 2013.

20. Gong Z, Zhao S, Zhou J, Yan J, Wang L, Du X, Li H, Chen Y, Cai $\mathrm{W}$ and $\mathrm{Wu}$ J: Curcumin alleviates DSS-induced colitis via inhibiting NLRP 3 inflammsome activation and IL- $1 \beta$ production. Mol Immunol 104: 11-19, 2018.

21. Ding XQ, Wu WY, Jiao RQ, Gu TT, Xu Q, Pan Y and Kong LD: Curcumin and allopurinol ameliorate fructose-induced hepatic inflammation in rats via miR-200a-mediated TXNIP/NLRP3 inflammasome inhibition. Pharmacol Res 137: 64-75, 2018

22. Han G, Wei Z, Cui H, Zhang W, Wei X, Lu Z and Bai X: NUSAP1 gene silencing inhibits cell proliferation, migration and invasion through inhibiting DNMT1 gene expression in human colorectal cancer. Exp Cell Res 367: 216-221, 2018.

23. Huang Y, An L, Hui KM, Ren Q and Wang W: An LDLa domain-containing C-type lectin is involved in the innate immunity of Eriocheir sinensis. Dev Comp Immunol 42: 333-344, 2014.

24. Livak KJ and Schmittgen TD: Analysis of relative gene expression data using real-time quantitative PCR and the 2(-Delta Delta C(T)) method. Methods 25: 402-408, 2001.

25. Goodman RB, Pugin J, Lee JS and Matthay MA: Cytokine-mediated inflammation in acute lung injury. Cytokine Growth Factor Rev 14: 523-535, 2003.

26. Wen SH, Wu HJ, Lin L, Chong L, Zhu LL, Zhang WX, Zhang HL and Li CC: Adjunctive dexamethasone therapy improves lung injury by inhibiting inflammation and reducing RIP3 expression during Staphylococcus aureus pneumonia in mice. Int Immunopharmacol 23: 709-718, 2014.

27. Chen Y, Nie YC, Luo YL, Lin F, Zheng YF, Cheng GH, Wu H, Zhang KJ, Su WW, Shen JG and Li PB: Protective effects of naringin against paraquat-induced acute lung injury and pulmonary fibrosis in mice. Food Chem Toxicol 58: 133-140, 2013.

28. Tyagi N, Kumari A, Dash D and Singh R: Protective effects of intranasal curcumin on paraquot induced acute lung injury (ALI) in mice. Environ Toxicol Pharmacol 38: 913-921, 2014.

29. Li GP, Yang H, Zong SB, Liu Q, Li L, Xu ZL, Zhou J, Wang ZZ and Xiao W: Diterpene ginkgolides meglumine injection protects against paraquat-induced lung injury and pulmonary fibrosis in rats. Biomed Pharmacother 99: 746-754, 2018.

30. Cheresh P, Kim SJ, Tulasiram S and Kamp DW: Oxidative stress and pulmonary fibrosis. Biochim Biophys Acta 1832: 1028-1040, 2013.

31. Das KC, Lewis-Molock Y and White CW: Elevation of manganese superoxide dismutase gene expression by thioredoxin. Am $\mathbf{J}$ Respir Cell Mol Biol 17: 713-726, 1997.

32. Hwang J, Suh HW, Jeon YH, Hwang E, Nguyen LT, Yeom J, Lee SG, Lee C, Kim KJ, Kang BS, et al: The structural basis for the negative regulation of thioredoxin by thioredoxin-interacting protein. Nat Commun 5: 2958, 2014

33. Yu X, Lan P, Hou X, Han Q, Lu N, Li T, Jiao C, Zhang J, Zhang C and Tian Z: HBV inhibits LPS-induced NLRP3 inflammasome activation and IL-1 $\beta$ production via suppressing the NF- $\kappa B$ pathway and ROS production. J Hepatol 66: 693-702, 2017. 
34. Dai J, Zhang X, Li L, Chen H and Chai Y: Autophagy inhibition contributes to ROS-producing NLRP3-dependent inflammasome activation and cytokine secretion in high glucose-induced macrophages. Cell Physiol Biochem 43: 247-256, 2017.

35. Heneka MT, Carson MJ, El Khoury J, Landreth GE, Brosseron F, Feinstein DL, Jacobs AH, Wyss-Coray T, Vitorica J, Ransohoff RM, et al: Neuroinflammation in Alzheimer's disease. Lancet Neurol 14: 388-405, 2015.

36. Masters SL, Dunne A, Subramanian SL, Hull RL, Tannahill GM, Sharp FA, Becker C, Franchi L, Yoshihara E, Chen Z, et al: Activation of the NLRP3 inflammasome by islet amyloid polypeptide provides a mechanism for enhanced IL- $1 \beta$ in type 2 diabetes. Nat Immunol 11: 897-904, 2010.

37. Wang Z, Hu W, Lu C, Ma Z, Jiang S, Gu C, Acuña-Castroviejo D and Yang Y: Targeting NLRP3 (Nucleotide-binding domain, leucine-rich-containing family, pyrin domain-containing-3) inflammasome in cardiovascular disorders. Arterioscler Thromb Vasc Biol 38: 2765-2779, 2018

38. Zhang M, Harashima N, Moritani T, Huang W and Harada M: The roles of ROS and caspases in TRAIL-induced apoptosis and necroptosis in human pancreatic cancer cells. PLoS One 10: e0127386, 2015.

39. Friedemann T, Otto B, Klätschke K, Schumacher U, Tao Y, Leung AK, Efferth T and Schroder S: Coptis chinensis franch. exhibits neuroprotective properties against oxidative stress in human neuroblastoma cells. J Ethnopharmacol 155: 607-615, 2014
40. Piggott K, Deng J, Warrington K, Younge B, Kubo JT, Desai M, Goronzy JJ and Weyand CM: Blocking the NOTCH pathway inhibits vascular inflammation in large-vessel vasculitis. Circulation 123: 309-318, 2011.

41. Poulsen LC, Edelmann RJ, Krüger S, Diéguezhurtado R, Shah A, Stavnoraas TE, Renzi A, Szymanska M, Wang J, Ehling M, et al: Inhibition of endothelial NOTCH1 signaling attenuates inflammation by reducing cytokine-mediated histone acetylation at inflammatory enhancers. Arterioscler Thromb Vasc Biol 38: 854-869, 2018

42. Singla RD, Wang J and Singla DK: Regulation of Notch 1 signaling in THP-1 cells enhances M2 macrophage differentiation. Am J Physiol Heart Circ Physiol 307: H1634-H1642, 2014.

43. Jiang L, Ke M, Yue S, Xiao W, Yan Y, Deng X, Ying QL, Li J and Ke B: Blockade of Notch signaling promotes acetaminophen-induced liver injury. Immunol Res 65: 739-749, 2017.

This work is licensed under a Creative Commons Attribution-NonCommercial-NoDerivatives 4.0 International (CC BY-NC-ND 4.0) License. 